\title{
Enhancement of Aedes aegypti susceptibility to dengue by Wolbachia is not supported
}

\author{
Thomas H. Ant ${ }^{1}$, Maria-Vittoria Mancini ${ }^{1}$, Julien Martinez (i) $^{1} \&$ Steven P. Sinkins (iD ${ }^{1 凶}$
}

ARISING FROM King et al. Nature Communications https://doi.org/10.1038/s41467-018-03981-8 (2018).

R eleases of Aedes aegypti mosquitoes carrying Wolbachia to block the transmission of dengue virus (DENV) are currently being deployed as a novel dengue control strategy in a number of countries, with very encouraging results ${ }^{1,2}$. In their paper entitled "Variation in Wolbachia effects on Aedes mosquitoes as a determinant of invasiveness and vectorial capacity", King et al. ${ }^{3}$ used DENV infection and transmission modelling to reinterpret experimental data from two previous studies ${ }^{4,5}$. The authors claimed that wMel Wolbachia increase the mean susceptibility of Ae. aegypti to DENV, contradicting various other studies $^{6-12}$. Here, we raise concerns with the experimental approaches used to generate one of the primary datasets on which the modelling is based, and we discuss how these limitations could make some of the original conclusions misleading.

King et al. ${ }^{3}$ base a large part of their modelling analysis on experimental data reported in Souto-Maior et al. ${ }^{4}$, consisting of intrathoracic challenges with DENV-1 of $w$ Mel-carrying and tetracycline-cured (TET) Ae. aegypti, with virus quantified by qRT-PCR in whole bodies. An equivalent proportion of denguepositive $w$ Mel-carrying mosquitoes compared to TET controls is reported at an injection titre of $10^{4} \mathrm{TCID} 50 / \mathrm{ml}$, a slight increase in positives in $w$ Mel-carrying mosquitoes at $10^{5}$ and $10^{6} \mathrm{TCID} 50 /$ $\mathrm{ml}$, and a slight decrease at $10^{7}$ and $10^{8} \mathrm{TCID} 50 / \mathrm{ml}$. Intrathoracic inoculation can be a convenient first approximation of infectivity since it is easier to perform than oral challenge; however it bypasses the biologically crucial midgut infection barrier, a key site of virus blocking by Wolbachia ${ }^{13}$. Thus, it represents a substantial deviation from the natural infection route, and its biological relevance is especially questionable given the binary presence/absence of viral RNA used by King et al. ${ }^{3}$ as the measure of susceptibility.

Furthermore, in Souto-Maior et al. ${ }^{4}$ a high proportion of the mosquitoes assigned as DENV-infected, particularly those at low virus titre challenges, are likely false positives. Examination of the raw qPCR cycle threshold $(\mathrm{Ct})$ values reveals a positive signal in 5 out of 35 negative control mosquitoes following mock challenge. The mean of these $\mathrm{Ct}$ values is lower, indicating a stronger signal, than many of the $\mathrm{Ct}$ values from mosquitoes counted as DENVinfected (Fig. 1a). A strong signal in the negative control leads to uncertainty over the specificity of the qPCR assay used and suggests that many of the readings indicating DENV-positivity from the $10^{4}, 10^{5}, 10^{6}$, and $10^{7}$ TCID $50 / \mathrm{ml}$ inoculations could be artefacts. The very low infection rates at the lower virus inoculation titres are similar to the false positive rate of the negative controls: $4 \% \quad(0.4-13.7 \%), \quad 8.8 \% \quad(1.8-23.7 \%)$, and $18.4 \%$ (8.4-30.9\%), for $10^{4}, 10^{5}$, and $10^{6} \mathrm{TCID} 50 / \mathrm{ml}$, respectively, compared to $14.3 \%(4.8-30.3 \%)$ for negative controls, where all percentage values in parentheses are exact binomial 95\% CI.

Despite a low number of virus-positive mosquitoes at the lower inoculation titres, with a total of 14 positive mosquitoes across both the $w \mathrm{Mel}$ and TET groups for the $10^{4}, 10^{5}$, and $10^{6}$ TCID50/ $\mathrm{ml}$ inoculations combined, resulting in low statistical power (Fig. 1b), King et al. ${ }^{3}$ fit a dose-response model to the data and conclude that "Wolbachia increase mean susceptibility [to infection] by a factor of 6.9". Even if all DENV-positive PCR readings are assumed to be valid, this increase in susceptibility is difficult to reconcile with the data. The total proportion of DENV-positive TET mosquitoes was 52 out of 117 or $44.4 \%$ (35.3-53.9\%) compared to 62 out of 143 or $43.3 \%$ (35.1-51.9) for $w \mathrm{Mel}$, a slight decrease in infection rate that was not statistically significant: $p=$ 0.96, Binomial test (Fig. 1c). There are also very low infection rates and infection titres in the Souto-Maior et al. ${ }^{4}$ dataset even at relatively high inoculation titres, e.g., an $8.3 \%$ infection rate in TET mosquitoes following DENV-inoculation at $10^{6} \mathrm{TCID} 50 / \mathrm{ml}$. This result is inconsistent with other studies: for example, $100 \%$ infection was observed following DENV inoculation at $10^{4}$ genome copies $/ \mathrm{ml}^{11} ; 95 \%$ infection following DENV-inoculation at $10^{5} \mathrm{TCID} 50 / \mathrm{ml}^{10}$; and $100 \%$ infection following DENV inoculation at $6 \times 10^{5} \mathrm{PFU} / \mathrm{ml}^{14}$. Finally, there are issues with data transfer, since King et al. ${ }^{3}$ present the $w$ Mel cohort as having a higher proportion DENV infected at $10^{4} \mathrm{TCID} 50 / \mathrm{ml}$, whereas in the Souto-Maior et al. ${ }^{4}$ dataset the proportions are equivalent.

The second dataset used by King et al. ${ }^{3}$ was from Ferguson et al. ${ }^{5}$, comparing $w$ Mel-carrying and Wolbachia-negative Ae. aegypti fed on viremic blood from dengue patients (serotypes 1-4). The study provides data on DENV infection rates and titres in dissected abdomens by qRT-PCR, and a direct assessment of the infectivity of mosquito saliva. Applying their dose-response modelling to proportions of infected abdomens, King et al. ${ }^{3}$ conclude an "increase in mean [ $w$ Mel-infected]

${ }^{1}$ Centre for Virus Research, University of Glasgow, Glasgow G12 8QQ, UK. ${ }^{凶}$ email: steven.sinkins@glasgow.ac.uk 

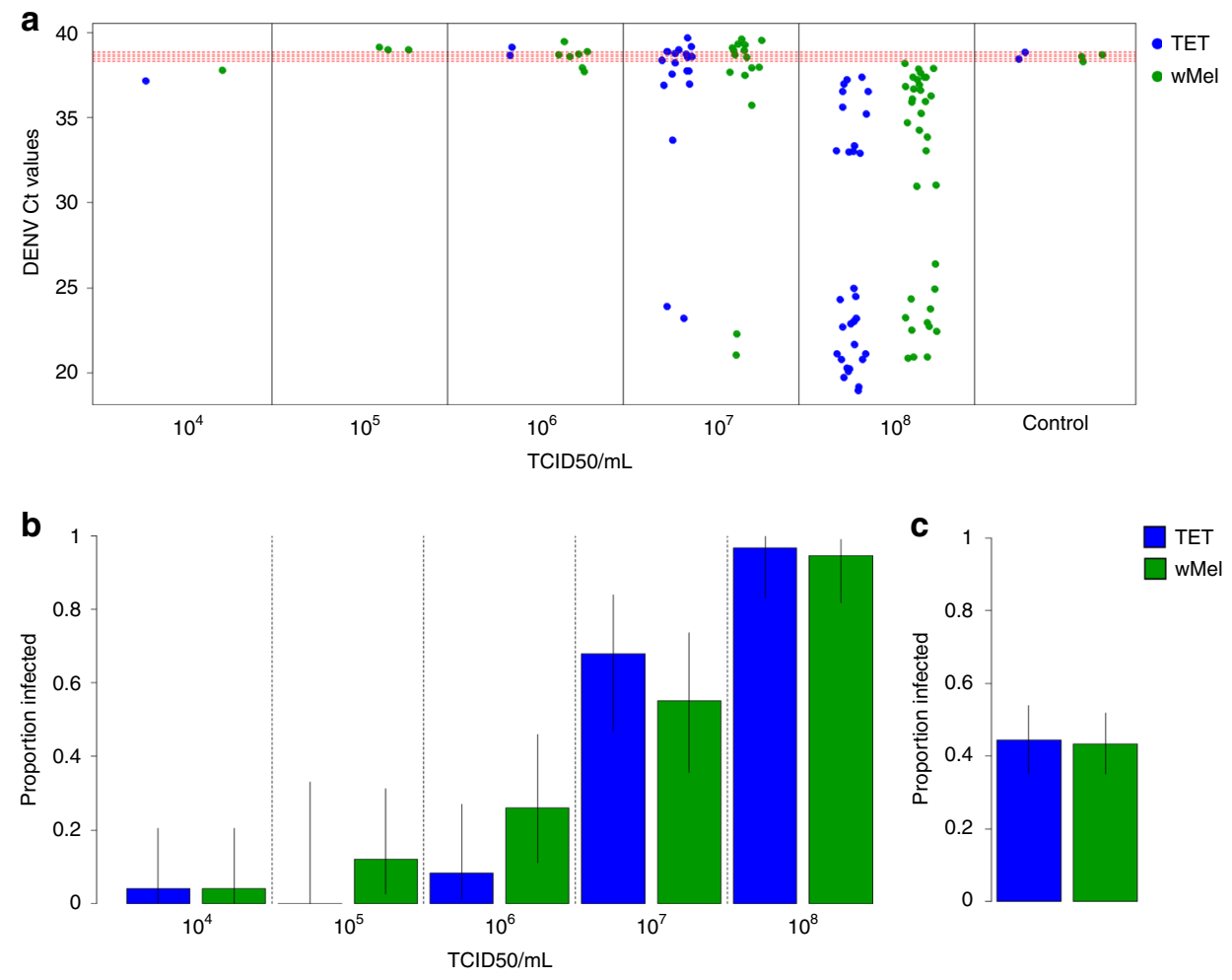

Fig. 1 qPCR data and DENV infection rates from the original Souto-Maior et al. ${ }^{\mathbf{4}}$ data. a Raw DENV qPCR Ct values resulting from intrathoracic inoculations. Blue and green dots show Ct values from TET and wMel mosquitoes, respectively. Red dashed lines show Ct values from mock-injected negative controls. b-c Proportions of TET and wMel mosquitoes classified by Souto-Maior et al. ${ }^{4}$ (and likewise by King et al. ${ }^{3}$ ) as qPCR positiveproportions have been recalculated from the original dataset. Error bars show $95 \%$ binomial confidence intervals.

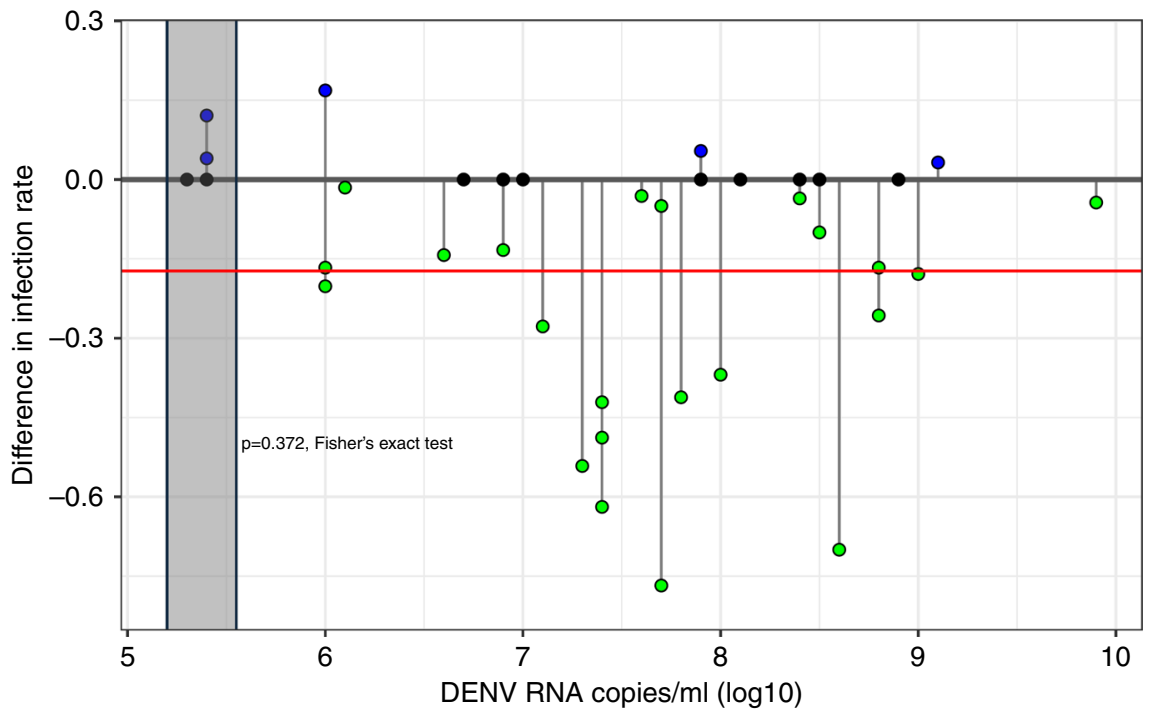

Fig. 2 Differences in abdomen infection rate [(rate in wMel)-(rate in Wolbachia-uninfected)] between wMel and Wolbachia-negative mosquitoes fed on viremic blood, replotted from the original Ferguson et al..$^{\mathbf{5}}$ data. Each dot represents a feeding on blood sourced from a different dengue-infected patient. Negative differences (green dots) indicate a lower abdomen infection rate in wMel-carrying mosquitoes. Positive differences (blue dots) indicate a higher abdomen infection rate in wMel-carrying mosquitoes. Black dots indicate no difference. The red line shows the mean difference across all DENV titres. Shaded area shows data points in the $10^{5} \leq \mathrm{X}<10^{6}$ RNA copies/ml stratum and statistical test.

mosquito susceptibility to infection to a factor of 1.5 " relative to Wolbachia-negative controls. However, the value is misleading. Replotting the Ferguson et al. ${ }^{5}$ abdomen infection rates (Fig. 2) shows that in 23 out of 42 feedings or $54.7 \%$ (38.67-70.2\%), $w$ Mel-carrying mosquitoes displayed a lower infection proportion than Wolbachia-negative controls; in 14 out of 42 or
$33.3 \%(19.6-49.6 \%)$ the proportions were exactly equivalent; and in only 5 out of 42 or $11.9 \%$ of feedings $(3.9-25.6 \%)$ was the proportion infected higher in $w$ Mel-carrying mosquitoes. Thus 665 out of a total 955 or $69.6 \%(66.6-72.5 \%)$ of Wolbachia-negative mosquitoes developed abdominal infections, compared to 507 out of a total 877 or $57.8 \%(54.4-61.1 \%)$ of 
$w$ Mel-carrying mosquitoes, a $16.9 \%$ decrease in infection rate: $p=0.0147$, Fisher's exact test.

In their susceptibility analysis, King et al. ${ }^{3}$ stratify the Ferguson et al..$^{5}$ abdomen data into groups of Log 10 viral titre i.e., patients with $10^{5} \leq \mathrm{X}<10^{6}$ and $10^{6} \leq \mathrm{X}<10^{7}$ RNA copies/ml etc. are grouped independently. Using this stratification system King et al. observe that there is an increased infection rate in the $w \mathrm{Mel}$ cohort in the $10^{5} \leq \mathrm{X}<10^{6}$ grouping. However, while 14 out of 75 or $18.7 \%(10.6-29.3 \%)$ of $w \mathrm{Mel}$ carriers were DENV positive in abdomens compared to 9 out of 80 or $11.3 \%(5.3-20.3 \%)$ of Wolbachia negatives, this difference is not statistically significant: $p=0.372$, Fisher's exact test (Fig. 2). At all other strata the proportion of infected mosquitoes was lower in the $w \mathrm{Mel}$ cohort. There was also no consideration of differences in output viral titres in challenged mosquitoes in the King et al. ${ }^{3}$ model; while in Ferguson et al. ${ }^{5}$ the $10^{5} \leq \mathrm{X}<10^{6}$ stratum shows a slight increase in infection rate for $w \mathrm{Mel}$, virus titre in the same mosquitoes is on average 10-100-fold less. The need for consideration of virus in the saliva is also paramount.

Evaluations of safety and efficacy of the Wolbachia replacement approach are valuable, but must be based on robust experimental foundations and analysis, given that misleading 'headline' statements can adversely impact stakeholder perceptions. Given low-titre asymptomatic carriers of DENV may be important contributors to its transmission ${ }^{15}$, the study by King et al. ${ }^{3}$ could even be interpreted as providing evidence of a risk that Wolbachia deployment could actually increase dengue incidence. Wolbachia replacement requires the release of biting female mosquitoes, and high levels of community support are thus essential; the intervention is particularly sensitive to any safety concerns. The limitations in the experimental dataset on which King et al. $^{3}$ based their analysis suggest that caution is needed when interpreting their argument for enhanced mean DENV susceptibility in Ae. aegypti carrying w Mel Wolbachia.

Reporting summary. Further information on research design is available in the Nature Research Reporting Summary linked to this article.

\section{Data availability}

There is no new data associated with this manuscript.

Received: 11 January 2020; Accepted: 27 October 2020;

Published online: 30 November 2020

\section{References}

1. Nazni, W. A. et al. Establishment of Wolbachia Strain wAlbB in Malaysian populations of Aedes aegypti for Dengue control. Curr. Biol. 29, 4241-4248 (2019). e4245.

2. Ryan, P. A. et al. Establishment of wMel Wolbachia in Aedes aegypti mosquitoes and reduction of local dengue transmission in Cairns and surrounding locations in northern Queensland, Australia. Gates Open Res. 3, 1547 (2019).

3. King, J. G., Souto-Maior, C., Sartori, L. M., Maciel-de-Freitas, R. \& Gomes, M. G. M. Variation in Wolbachia effects on Aedes mosquitoes as a determinant of invasiveness and vectorial capacity. Nat. Commun. 9, 1483 (2018).

4. Souto-Maior, C., Sylvestre, G., Braga Stehling Dias, F., Gomes, M. G. M. \& Maciel-de-Freitas, R. Model-based inference from multiple dose, time course data reveals Wolbachia effects on infection profiles of type 1 dengue virus in Aedes aegypti. PLoS Negl. Trop. Dis. 12, e0006339 (2018).
5. Ferguson, N. M. et al. Modeling the impact on virus transmission of Wolbachia-mediated blocking of dengue virus infection of Aedes aegypti. Sci. Transl. Med. 7, 279ra237 (2015).

6. Ant, T. H., Herd, C. S., Geoghegan, V., Hoffmann, A. A. \& Sinkins, S. P. The Wolbachia strain $w$ Au provides highly efficient virus transmission blocking in Aedes aegypti. PLoS Pathog. 14, e1006815 (2018).

7. Walker, T. et al. The $w \mathrm{Mel}$ Wolbachia strain blocks dengue and invades caged Aedes aegypti populations. Nature 476, 450-453 (2011).

8. Frentiu, F. D. et al. Limited dengue virus replication in field-collected Aedes aegypti mosquitoes infected with Wolbachia. PLoS Negl. Trop. Dis. 8, e2688 (2014).

9. Moreira, L. A. et al. A Wolbachia symbiont in Aedes aegypti limits infection with dengue, Chikungunya, and Plasmodium. Cell 139, 1268-1278 (2009).

10. Fraser, J. E. et al. Novel Wolbachia-transinfected Aedes aegypti mosquitoes possess diverse fitness and vector competence phenotypes. PLoS Pathog. 13, e1006751 (2017).

11. Joubert, D. A. et al. Establishment of a Wolbachia superinfection in Aedes aegypti Mosquitoes as a potential approach for future resistance management. PLoS Pathog. 12, e1005434 (2016).

12. Bian, G., Xu, Y., Lu, P., Xie, Y. \& Xi, Z. The endosymbiotic bacterium Wolbachia induces resistance to dengue virus in Aedes aegypti. PLoS Pathog. 6, e1000833 (2010).

13. Chouin-Carneiro, T. et al. Wolbachia strain $w$ AlbA blocks Zika virus transmission in Aedes aegypti. Med. Vet. Entomol. https://doi.org/10.1111/ mve.12384 (2019).

14. Pacidonio, E. C., Caragata, E. P., Alves, D. M., Marques, J. T. \& Moreira, L. A. The impact of Wolbachia infection on the rate of vertical transmission of dengue virus in Brazilian Aedes aegypti. Parasit. Vectors 10, 296 (2017).

15. Duong, V. et al. Asymptomatic humans transmit dengue virus to mosquitoes. Proc. Natl Acad. Sci. USA 112, 14688-14693 (2015).

\section{Author contributions}

T.A., M.V.M., J.M., and S.P.S. wrote the manuscript.

\section{Competing interests}

The authors declare no competing interests.

\section{Additional information}

Supplementary information is available for this paper at https://doi.org/10.1038/s41467020-19830-6.

Correspondence and requests for materials should be addressed to S.P.S.

Peer review information Nature Communications thanks Zhiyong Xi and the other, anonymous, reviewer for their contribution to the peer review of this work.

Reprints and permission information is available at http://www.nature.com/reprints

Publisher's note Springer Nature remains neutral with regard to jurisdictional claims in published maps and institutional affiliations.
Open Access This article is licensed under a Creative Commons Attribution 4.0 International License, which permits use, sharing, adaptation, distribution and reproduction in any medium or format, as long as you give appropriate credit to the original author(s) and the source, provide a link to the Creative Commons license, and indicate if changes were made. The images or other third party material in this article are included in the article's Creative Commons license, unless indicated otherwise in a credit line to the material. If material is not included in the article's Creative Commons license and your intended use is not permitted by statutory regulation or exceeds the permitted use, you will need to obtain permission directly from the copyright holder. To view a copy of this license, visit http://creativecommons.org/ licenses/by/4.0/

(C) The Author(s) 2020 